Portland State University

PDXScholar

\title{
A Paradigm Shift: From a Categorical to Dimensional Diagnostic Model of Personality Disorder
}

Jessica Green

Portland State University

Follow this and additional works at: https://pdxscholar.library.pdx.edu/honorstheses

Let us know how access to this document benefits you.

\section{Recommended Citation}

Green, Jessica, "A Paradigm Shift: From a Categorical to Dimensional Diagnostic Model of Personality Disorder" (2015). University Honors Theses. Paper 190.

https://doi.org/10.15760/honors.178

This Thesis is brought to you for free and open access. It has been accepted for inclusion in University Honors Theses by an authorized administrator of PDXScholar. Please contact us if we can make this document more accessible: pdxscholar@pdx.edu. 
A Paradigm Shift: From a Categorical to Dimensional Diagnostic Model of Personality Disorder

By

Jessica Green

An undergraduate honors thesis submitted in partial fulfillment of the

\author{
requirements for the degree of \\ Bachelor of Science \\ in \\ University Honors \\ and \\ Psychology
}

Thesis Adviser

Tina Burdsall

Portland State University

2015 


\begin{abstract}
This thesis discusses the possibility of a paradigm shift in the conceptualization of personality disorders (PDs), and eventually all psychological disorders, from categorical to dimensional. It examines the three main types of models utilized for diagnosing PDs. These main types are: the categorical model, where symptoms are organized in a check list based on categories; the dimensional model, where symptoms are organized on a spectrum rather than in a list; and the hybrid model, which is a combination of the two. It focuses on the strengths and weaknesses of each model and how they are used to define and diagnose PDs. In conclusion, there are significant gaps in the empirical evidence pertaining to the practical applications of the dimensional and hybrid models, therefore, a change in diagnostic criteria is not yet advised. Only when these gaps have been filled can a paradigmatic shift from a categorical to a dimensional conceptualization of PDs, and eventually all psychological disorders, occur.
\end{abstract}




\section{Table of Contents}

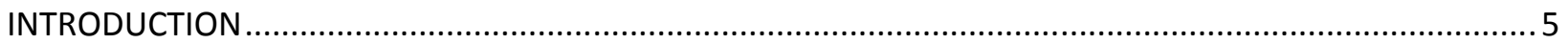

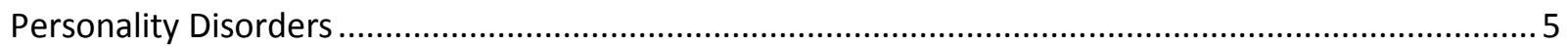

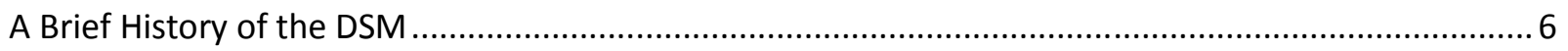

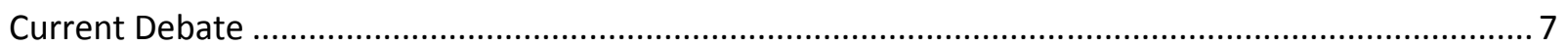

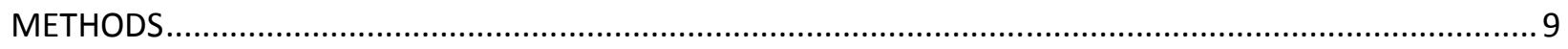

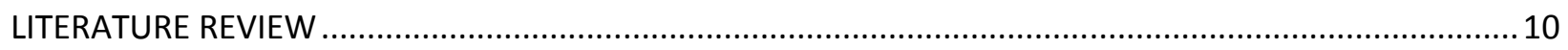

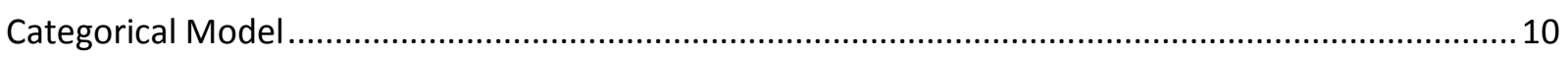

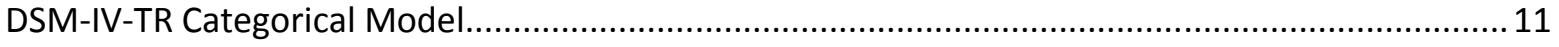

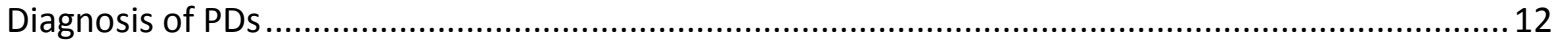

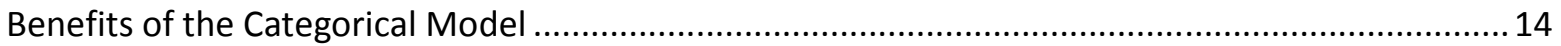

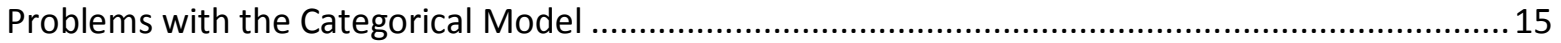

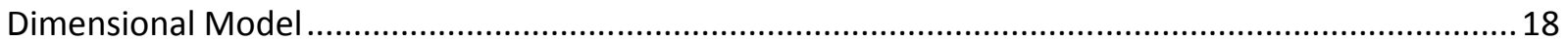

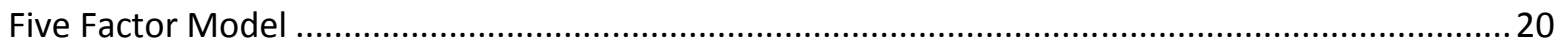

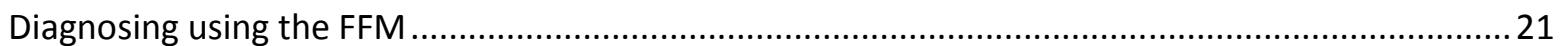

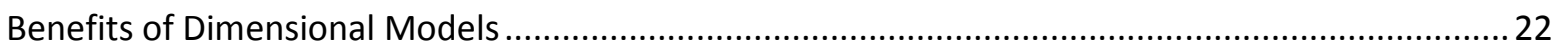

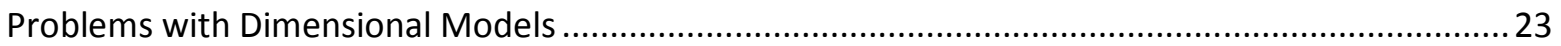

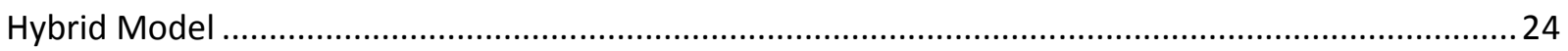

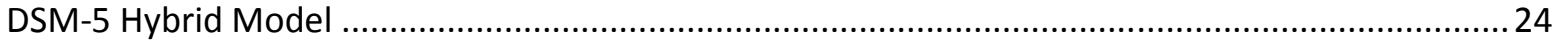

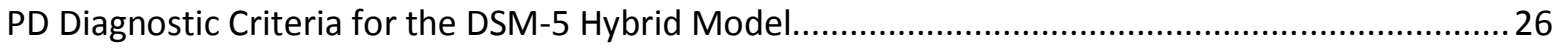




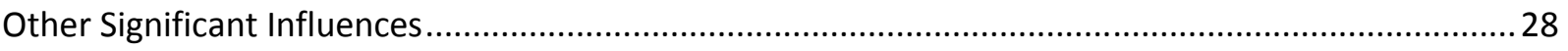

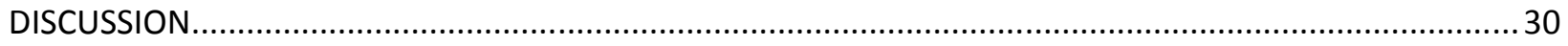

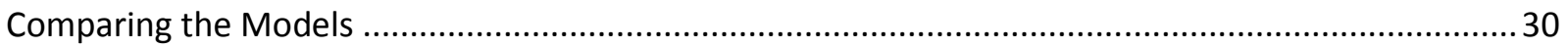

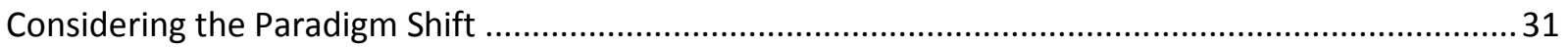

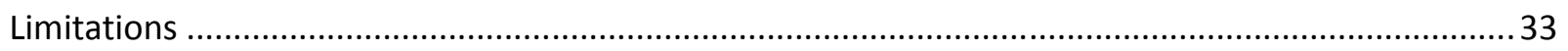

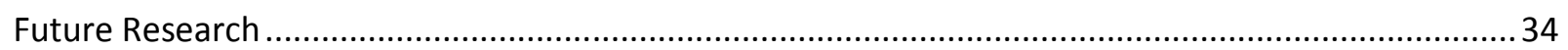

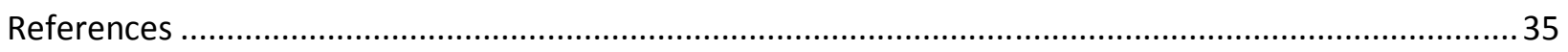




\section{INTRODUCTION}

The psychological community is on the brink of a paradigm shift in the way mental disorders are conceptualized. This paradigm shift can be seen as a change in the way mental disorders are described and their diagnostic criteria. Personality Disorders (PDs) are on the forefront of this transition. Support for a change in the diagnostic criteria from a categorical, or check list, diagnostic model to a dimensional, or scale, diagnostic model is accumulating as the categorical model is no longer the most effective or efficient way to describe and diagnose PDs. However, there are barriers preventing this change from occurring and these barriers must be addressed so the necessary changes in the diagnostic criteria for PDs can occur.

\section{Personality Disorders}

A personality disorder (PD) is "an enduring pattern of inner experience and behavior that deviates markedly from the expectations of the individual's culture, is pervasive and inflexible, has an onset in adolescence or early adulthood, is stable over time, and leads to distress or impairment" (APA, pg 629, 2000). In other words a PD is a pattern of behaviors consistently expressed by individuals that cause problems in their lives. By this definition, PDs are seen as qualitatively distinct conditions. This view is similar to how most physical disorders are seen, where the disorder is described and diagnosed based on the expressed symptoms of the patient (Widiger \& Mullins-Sweatt, 2010). For example, a person who holds a grudge, is unforgiving, and is constantly suspicious of others might qualify for a diagnosis of Paranoid PD which can cause severe impairment in a person's ability to create and maintain social relationships. PDs are more prevalent than is realized by society. Chances are, everyone is related to, or knows someone related to, a person suffering from a PD. Reports state that $15-20 \%$ of the general 
public suffers from one or more personality disorders (APA, 2000). However, this percentage reflects the number of individuals who sought treatment and received a diagnosis for a PD; it does not include the individuals who do not seek help but are afflicted with a PD. Research shows that PDs are associated with reduced quality of life and dysfunction in almost every area with which health care providers are concerned (Clark, 2007) and therefore should be accorded the most effective and efficient diagnostic model and treatment options possible.

\section{A Brief History of the DSM}

Descriptions and diagnostic criteria for all psychological disorders (e.g., Schizophrenia, Mood Disorders, and PDs) can be found in a single manual called the Diagnostic and Statistical Manual of Mental Disorders or DSM. This is the manual most clinicians use when diagnosing PDs and all other psychological disorders. The American Psychological Association (APA) published the first edition of the DSM in 1952 as a standardized way for clinicians to diagnose mental disorders and to improve communication among mental health professionals (Blashfield et al., 2014). The most recent edition of the DSM was published in May 2013 and is the fifth edition (DSM-5). A categorical model, where symptoms are organized in a check list based on categories, was first utilized for diagnostics in the third edition of the DSM that was published in 1980 (Krueger et al., 2014). The PD diagnostic model in the DSM-5 is the same categorical model used in the DSM-IV-TR (Diagnostic and Statistical Manual of Mental Disorders fourth edition text revision), in the DSM-III-R, and in the DSM-III. In fact, the categorical model has not changed significantly since it was originally published in the DSM-III in 1980 (Krueger et al., 2014) even though clinicians have encountered multiple problems using this model. However, the Board of Trustees of the American Psychiatric Association (APA) has included a second PD 
model they named an alternative hybrid model of PDs in Section III of the DSM-5. This section is where the APA place emerging models that they believe need more research. The DSM-5 hybrid model is different from traditional rubrics because it was developed based on empirical research when, typically, models had been developed based on clinical authority (Krueger et al., 2014; Krueger \& Markon, 2014), meaning that criteria or symptoms were selected based on the beliefs of the more distinguished clinicians instead of on empirical research. This hybrid model is the first empirically-based maladaptive trait model to be featured in the DSM (Krueger \& Markon, 2014). A maladaptive trait model is a model based on behavioral traits that cause problems or dysfunction in an individual's life.

\section{Current Debate}

The method of PD diagnosis is currently a major topic of debate among researchers and clinicians within the psychological community (Morey et al., 2014). There are three main types of models for describing and diagnosing PDs that are currently being researched. These differ based on their structure and their diagnostic criteria (i.e., their symptoms). Some experts claim that the current categorical model is the most efficient and accurate method of diagnosis while others believe that a dimensional model, where symptoms are organized on a spectrum rather than in a list, would be more descriptive and accurate. Still others argue that a hybrid model would be most effective. All three of these models have strengths and weaknesses; however, currently, there is not enough empirical evidence to determine if one model works better than the others for diagnostics.

The relative merits of categorical and dimensional approaches to PD have been debated since the DSM-III was released in 1980 (Skodol, 2012). The categorical approach is currently the 
approach used by clinicians and has been since its original publication in 1980; however, empirical evidence has been collecting for decades showing that the structure of PDs can better be described using a dimensional approach (Clark, 2007). One survey reports that $74 \%$ of experts think the DSM-IV-TR categorical model of PDs should be replaced, $87 \%$ believe personality pathology is dimensional in nature, and 70\% indicated that a hybrid model of PDs is the most desirable alternative to the categorical model (Morey et al., 2014). The DSM-5 is the first edition of the DSM to include an empirically based model of maladaptive personality traits (Krueger \& Markon, 2014). The main reason it has taken this long to adopt the empirically supported dimensional PD structure is due to the numerous competing dimensional models referenced in the literature (Wright et al., 2012), including the Five Factor Model (FFM), the Schedule for Non-adaptive and Adaptive Personality (SNAP) model, and the Cloninger's Temperament and Character Inventory. These models differ from one another based on what traits they measure and what tools they use to measure those traits. Each model has its own set of advantages and disadvantages. Many professionals believe it is not a matter of whether the shift to a dimensional model will occur but when it will occur and which model will be chosen (Clark, 2007).

A shift in the DSM diagnostic model for PDs would begin a paradigmatic change in the conceptualization of all psychological disorder (Tackett et al., 2009). The change in conceptualization will alter the way that all mental disorders are described and diagnosed from a categorical to a dimensional approach, and this modification can be initiated with PDs. This transition depends on a diagnostic change within the DSM, if the model employed by the DSM does not change then the paradigm shift will not occur.

This thesis will argue that theoretically, a dimensional model is the foremost model for describing and diagnosing PDs when compared to the categorical and hybrid models; however, 
there are multiple barriers that must be addressed before a paradigm shift can occur. One barrier are the gaps in the research, including the practical applications of the dimensional and hybrid models, the effect of these models on the stigmatization and labeling issues of the categorical model, and how these new models will interact with insurance companies that provide coverage for treatment. Another barrier is the resistance to change among some clinicians. In the following sections of this thesis, each of the three main diagnostic models of PDs will be thoroughly examined along with other factors that influence the selection of a diagnostic model including interactions with insurance companies and the control exercised by the APA when they decide what is published in the DSM.

\section{METHODS}

The research articles used in this examination were gathered using the PsychINFO database and GoogleScholar through Portland State University Library. All the cited articles pertaining to diagnostic categories were published between 2003 and 2014, with the majority of the articles being published between 2010 and 2014. These date limitations were set to gather the most recent research available. A search within these parameters was conducted using keywords such as personality disorder, DSM-5, diagnostic criteria, categorical model, dimensional model, and hybrid model. Articles were then selected based on content and their relevance to the topic. Some articles were selected because of their high prevalence in the references of the research articles already selected, those articles were then located through PsychINFO or GoogleScholar.

The DSM-5 was published in May, 2013 but it retained the same categorical model of PDs used in the DSM-IV-TR. For the purposes of this thesis, the DSM-IV-TR, which was published by the APA in 2000, is used because it is the edition that is either used or referenced 
by the majority of the research. The insurance articles referenced in this thesis were located in the same manner as the research articles and the articles pertain to both the DSM-IV-TR and the DSM-5 because both are referenced in this thesis.

\section{LITERATURE REVIEW}

In the following sections, the categorical model, the dimensional model, and the hybrid model of PDs are examined. The diagnostic criteria and process of diagnoses for each model are explained and their clinical strengths and weaknesses analyzed. Given that diagnostic models are not the only influence on the occurrence of a paradigm shift, other influences such as insurance coverage and who makes the final decisions concerning changes to the DSM are examined after the different diagnostic models.

\section{Categorical Model}

The categorical model of PDs is based on the medical disease model, meaning that examining the majority of an individual's symptoms leads to the diagnosis (Krueger et al., 2014). With this type of model there are only two possible conclusions: an individual has a disorder, or they do not have a disorder (Krueger et al., 2014). For example, a child covered in red spots that

itch either has the chicken pox or they do not; in this case the specific symptoms of red spots and itching would lead to a diagnosis of chicken pox. It is a black and white model where symptoms directly lead to a diagnosis (e.g., chicken pox). 


\section{DSM-IV-TR Categorical Model}

The DSM-IV-TR is the edition of the DSM referenced in the following sections.

Although it is not the most recent edition of the DSM, the categorical model found in the DSMIV-TR was reprinted almost verbatim in Section II of the DSM-5 (Krueger et al., 2014). Five Axes were utilized to organize the DSM-IV-TR. Axis I refers broadly to a principal disorder that requires immediate attention. Axis II lists any and all PDs that may be relevant to the patient and any developmental disorders. Relevant medical or neurological conditions are listed in Axis III while Axis IV lists codes for major psychological stressors the individual may have faced recently. Axis V includes codes for the level of function the individual can attain (APA, 2000). The categorical model used for diagnosing PDs is found within Axis II. In the DSM-IV-TR, personality traits are specifically used to diagnose personality disorders and are defined as an "enduring pattern of perceiving, relating to, and thinking about the environment and oneself that is exhibited in a wide range of social and personal contexts" (APA, pg 630, 2000). A personality trait must be inflexible, maladaptive, persisting and cause significant functional impairment or subjective distress to the individual for it to be regarded as a symptom of a personality disorder and used in the diagnosis of the disorder.

The DSM-IV-TR includes ten specific personality disorders which are grouped into three clusters based on descriptive similarities. Cluster A is defined by individuals with odd or eccentric behaviors. It includes the Paranoid, Schizoid, and Schizotypal PDs. Cluster B is defined by dramatic, emotional, or erratic behaviors and includes the Antisocial, Borderline, Histrionic, and Narcissistic PDs. Finally, Cluster C includes Avoidant, Dependent, and Obsessive-Compulsive PDs and is defined by anxious or fearful behaviors. An additional PD category exists as a nondescript category for individuals that are considered to be suffering from 
a PD but do not meet the criteria for any specific PD category. It is called PD-NOS or Personality Disorder-Not Otherwise Specified. An individual that meets the criteria for a general $\mathrm{PD}$, which a patient diagnosed with a any PD is required to meet, but does not meet the criteria for a specific PD would be given this diagnosis. PD-NOS is the most frequently used PD diagnosis (Skodol, 2012).

\section{Diagnosis of PDs}

There are two steps when diagnosing an individual using the DSM-IV-TR diagnostic criteria. The individual should meet the minimum criteria for a general PD diagnosis, and they should also meet the criteria for a specific PD. An individual must exhibit six criteria to be diagnosed with a general personality disorder. These criteria, A-F, describe the necessary requirements for a general PD diagnosis including problems related to their expressed behaviors, time constraints, and exclusions. A person must exhibit an enduring pattern of experiences and behaviors that are markedly different from the expectations of their culture in at least two of the following areas: cognition (i.e., thinking), affectivity or expressing emotion, interpersonal functioning, and impulse control (Criterion A). These experiences and behaviors need to be inflexible and pervasive across a broad range of personal and social situations (Criterion B) and they must lead to clinically significant distress or impairment in social, occupational, or other areas of functioning (Criterion C). The pattern should be stable and last for a long period of time, with its onset in early adulthood or adolescence (Criterion D). The pattern cannot be a manifestation or consequence of another mental disorder (Criterion E) and it cannot be due to the physiological effects of substances (such as drugs) or a general medical condition like head 
trauma (Criterion F) (APA, 2000). If an individual meets all of these criteria he/she is diagnosed with a general PD.

Each of the ten PDs have different criteria that must be met, but they all follow the same general format. For example, Paranoid PD is defined as "a pattern of pervasive distrust and suspiciousness of others such that their motives are interpreted as malevolent" (APA, pg 634, 2000) and is indicated by the presence of at least four of the following seven criteria: (1) suspecting without enough evidence that others are exploiting, harming, or deceiving them; (2) preoccupation with unjustified doubts about the loyalty or trustworthiness of friends or associates, (3) the reluctance to confide in others because of a needless fear of the information being used maliciously against them; (4) misinterpreting hidden meanings, demeaning or threatening, in benign remarks or events; (5) persistently bearing grudges (i.e., unwillingness to forgive); (6) perceiving attacks on their character or reputation that others do not see and responding quickly in anger or to counterattack; (7) having constant suspicions, without justification, about the fidelity of their spouse or sexual partner. These patterns must occur outside the course of a separate disorder (e.g., Schizophrenia) and cannot be seen only as a result of a medical condition (e.g., head trauma). The APA ordered these criteria by what they believed to be decreasing diagnostic importance meaning criterion 1 is more important to the diagnosis than criterion 7 although no specific criterion is required for a diagnosis. The diagnostic importance was measured by data on the diagnostic efficiency of each criterion when available (APA, 2000). This efficiency included how well each criterion predicted the existence of the disorder and how prevalent the criterion was in the people diagnosed with the disorder.

An individual's diagnosis is typically based on a combination of three things: an interview with his/her clinician, behavior observations by his/her clinician, and a mental status 
exam consisting of an assessment of aspects of his/her mental functioning (Sprock, 2003). A single interview is all that is actually required and can be enough to make a diagnosis. Several interviews spread out over time, however, along with additional supplemental materials, such as observations of behaviors and mental status exams, is the method of diagnosis preferred by most clinicians (Sprock, 2003).

\section{Benefits of the Categorical Model}

One of the main benefits of the categorical model is its high ratings on clinical utility (Morey et al., 2014; Sprock, 2003; Widiger \& Mullins-Sweatt, 2010). Clinical utility is defined as the extent to which clinicians are assisted by the DSM when performing their various duties. It is operationalized by ease of use, communication, and treatment planning (Morey et al., 2014; Sprock, 2003; Widiger \& Mullins-Sweatt, 2010). The categories of this model are familiar to clinicians (Samuel et al., 2011; Sprock, 2003) because they have been using them unaltered from the time when the DSM-IV-TR was published in 1994 (Morey et al., 2014). This familiarity makes them easy to use. The PD system remained essentially unchanged from the first categorical model found in the DSM-III, published in 1980 (Krueger et al., 2014). Communication between professionals, and between clinician and patient, is clear, simple and efficient (Morey et al., 2014). With the existing categories there are obvious implications for treatment decisions. A clinician selects a treatment from a range of options that are considered appropriate based on the patient's categorical PD diagnosis. If the treatment works, it is followed to completion. If not, either a new treatment is chosen or the diagnosis is reassessed (Widiger \& Mullins-Sweatt, 2010). Other benefits of the categorical model include high interrater reliability and high clinical confidence (Sprock, 2003). In other words, clinicians are confident in their 
clinical diagnoses when using the DSM-IV-TR PD model and the diagnoses are reliable across different clinicians (i.e., multiple clinicians reached the same diagnosis). It has been suggested that clinicians prefer the categorical classification system based on these many benefits (Shedler et al., 2010; Sprock, 2003).

\section{Problems with the Categorical Model}

The categorical diagnostic model of PDs used in the DSM-IV-TR has multiple problems. This system defines PDs as qualitatively distinct clinical syndromes (APA, 2000) and the majority of the difficulties clinicians face using this model stem from reality contradicting this definition. The DSM-IV-TR has poor convergent and discriminant validity (Anderson et al., 2014; Morey et al., 2014). Convergent validity refers to how accurately a specific criterion predicts the PD category it describes, and discriminant validity refers to how that criterion does not predict the remaining PD categories. For example, the criterion of holding a grudge should predict a diagnosis of Paranoid PD (convergent validity) and it should not predict a diagnosis of obsessive-compulsive PD (discriminant validity).

Temporal instability, or changes over time, of symptoms is also a struggle the DSM-IVTR model encounters (Anderson et al., 2014; Morey et al., 2014; Wright et al., 2012). PDs are defined as disorders that are stable over time, therefore clinicians struggle when the symptoms expressed by a patient change usually due to the beginning or ending of treatment. The overarching disorder may remain the same while the defining symptoms shift as time passes and clinicians struggle to accommodate this type of change because it contradicts the definition provided in the DSM. 
A lack of variation within diagnoses and the discrepancies among patients are other problems faced by the DSM-IV-TR model (Anderson et al. 2014; Krueger et al., 2014; Morey et al., 2014; Wright et al., 2012). The lack of diagnostic variation stems from gathering individuals into categories, and a failure to appreciate the considerable variety among patients (Widiger \& Mullins-Sweatt, 2010). This is an issue because the criteria is polythetic, meaning that criteria may be shared among patients but no criterion is absolutely required for diagnosis, and a multiplicity of criteria combinations justify the same diagnosis. For example, there are 256 possible combinations of criteria in the DSM-IV-TR that qualify for a diagnosis of borderline PD which corresponds to an extreme amount of variation among patients with the same disorder (Skodol, 2012).

Another problem of the categorical model is the excessive rates of diagnostic comorbidity. Comorbidity is a term typically used to describe two things occurring simultaneously. However, PD experts use it to indicate when simultaneous diagnoses occur at a greater than chance rate (Krueger et al., 2014). Patients are being diagnosed with multiple PDs more often than they are diagnosed with a single PD. This means that the individual may not actually be suffering from multiple PDs even if they were diagnosed with multiple disorders. Reported rates of comorbidity within Axis II disorders are 50\% or greater in clinical samples (Clark, 2007), meaning half of the patients who are diagnosed with multiple disorders do not actually have multiple disorders. Those rates correspond to the average number of PDs diagnosed per patient in clinical samples with a range of 2.8 to 4.6 (Krueger et al., 2014), which indicates that clinical patients are diagnosed, on average, with approximately 3-5 PDs at one time. This over diagnosis leads to ineffective communication between all parties and non-specific treatment options which are less effective (Widiger \& Mullin-Sweatt, 2010). 
The DSM-IV-TR defines PDs as qualitatively distinct clinical syndromes that are organized into three clusters based on descriptive similarities (APA, 2000). However, there is no empirical evidence for distinct boundaries between the different PD categories; leading to high rates of co-occurrence which is when multiple disorders occur at once. This is another substantial issue with the categorical model of PDs (Grant et. al 2005; Skodol, 2012; Widiger \& MullinSweatt, 2010). Specific diagnoses lead to more effective treatments but categorical diagnoses are not very specific. The distinctions between PD categories are arbitrary cutoffs with little or no empirical support (Morey et al., 2014; Wright et al., 2012). There is also a lack of supporting empirical evidence for the number of criteria required to obtain a specific PD diagnosis. In the case of the previous example, there is no evidence that demonstrates why four out of the seven criteria are required for a paranoid PD diagnosis rather than three or five (Skodol, 2012).

Not only is there a high rate of comorbidity and co-occurrence among PD categories, but many patients who are diagnosed with a PD and also desire treatment, fail to meet enough of the listed criteria for a specific PD diagnosis (Arbisi, 2014). These patients frequently received a diagnosis of PD-NOS (Arbisi, 2014) which, as mentioned earlier, is used as a catch all for individuals who express the general criteria for having a PD but do not fit into a specific category. The lack of diagnostic specificity, and subsequent lack of treatment specificity, is the rule instead of the exception (Widiger \& Mullins-Sweatt, 2010). Specific treatment plans are more successful at improving patient impairment. Communication between professionals is difficult when trying to convey an individual patient's dysfunction because many patients are not adequately described by any of the ten PD categories included in the DSM-IV-TR or they are described by multiple categories simultaneously (Arbisi, 2014). Diagnoses using a categorical 
model have been called arbitrary, often unreliable, overlapping, and incomplete with only a limited utility for treatment planning (Clark, 2007).

There have also been reported problems with stigmatization of patients when using a categorical diagnostic model. This is important because people are often negatively impacted by stigmas (Hinshaw, 2006). The term stigma originates from the historical practices of physically branding individuals of chastised groups so they bore a visible sign of disgrace. To be stigmatized in the present is to be devalued by society based on characteristics an individual possesses. Stigmas can lead to stereotypes, fear, and rejection of individuals by society and these circumstances can be very detrimental to those individuals (Hinshaw, 2006) because humans are social being and individuals develop and change based on social interactions. The labels associated with categorical model PD diagnoses readily lead to stigmatized behavioral problems being associated with the individuals diagnosed with PDs. Stigmas can cause internal turmoil as well, due to the egosyntonic nature of PDs. Egosyntonic refers to the aspects of an individual's thoughts and behaviors that are acceptable and consistent with his/her self-concept. A PD diagnosis suggests that the individual's problem is who they are and who they always have been: that their identity is their mental disorder (Widiger \& Mullins-Sweatt, 2010). To imply that a person's personality or identity is the disorder causing their problems suggests that it is their own fault they are suffering. It is a form of blaming the victim and has adverse effects on their chances of recovery.

\section{Dimensional Model}

From a dimensional perspective, PDs are a collection of maladaptive personality traits that can be defined quantitatively instead of qualitatively as they are in the categorical model. 
(Widiger \& Mullins-Sweatt, 2010). There are numerous dimensional models for diagnosing PDs that implement a wide variety of diagnostic criteria. For example, the Five Factor Model (FFM), the Schedule for Non-adaptive and Adaptive Personality (SNAP) model, and the Cloninger's Temperament and Character Inventory are all dimensional models that are vastly different in their appearance and their diagnostic criteria. The FFM has been the primary focus of dimensional research and is the predominant dimensional model among professionals (Morey et al., 2014; Sprock, 2003). The SNAP model consists of 15 scales of measurement, twelve trait scales and three general temperament scales, that are indicative of personality pathology.

Mistrust, self-harm, and aggression are examples of the trait scales while the temperament scales are negative temperament, positive temperament, and disinhibition. The SNAP measurement was designed to assess an individual's personality characteristics within normal and abnormal ranges and to identify the underlying core dimensions of PDs (Morey et. al., 2007). These core dimensions are the twelve trait scales and the three temperament scales that this model measures. The Cloninger's Temperament and Character Inventory is a psychobiological model that consists of four temperament dimensions based on nuerobiochemical motivational systems and three characterological dimensions that are environmentally based (Sprock, 2003). The neurobiochemical basis for the temperament dimensions are identified as novelty seeking, harm avoidance, reward dependence, and persistence. The characterological dimensions are described as self-directedness, cooperativeness, and self-transcendence (i.e., experiencing spiritual ideas). Individuals who suffer from PDs typically rate low on these character dimensions so the nature of their disorder is determined by their temperament dimensions (Sprock, 2003). 


\section{Five Factor Model}

The Five Factor Model (FFM) is the most prominent dimensional model (Sprock, 2003) and is a measure of personality pathology based on the Big 5 model of personality which describes an individual's Openness to Experience, Conscientiousness, Extraversion, Agreeableness, and Neuroticism and is the most used measure for normal non-pathological personality (Morey et al., 2007). Within the FFM, PDs represent extreme or maladaptive variants of normal personality traits (Morey et al., 2007). It incorporates normal personality characteristics with abnormal characteristics to give a measure of the individual's whole personality.

As mentioned earlier, the FFM is currently the most researched dimensional model. There are five broad domains in the FFM and six specific facets to describe and measure each domain. The five broad domains consist of spectral scales of two extremes; they are: neuroticism vs emotional stability, extraversion vs introversion, openness vs closedness to experience, agreeableness vs antagonism, and conscientiousness (i.e., being thorough, careful, and vigilant) vs disinhibition (i.e., lack of restraint or impulsivity) (Widiger \& Mullins-Sweatt, 2010). The two extremes of each domain are mutually exclusive, if an individual is high on extraversion then they are low on introversion, they cannot be high on both extremes. An individual will be placed along the spectrum based on the six facets of each domain. For example, the six facets of agreeableness vs antagonism are: trust vs mistrust, straightforward vs deception, altruism (i.e., selflessness) vs self-centeredness, compliance vs opposition, modesty vs self-assurance, and tender-mindedness vs tough-mindedness. If an individual measured high in trust, straightforwardness, self-centeredness, compliance, modesty, and tender-mindedness he/she 
would fall significantly closer to the agreeableness end of the domain spectrum, even though self-centeredness is more indicative of antagonism.

\section{Diagnosing using the FFM}

There is a four step procedure for diagnosing PDs using the FFM to which the majority of clinicians and researchers adhere (Samuel et al., 2011; Widiger \& Mullins-Sweatt, 2010). Step one is to describe the individual's general personality structure in terms of the FFM using the five domains and all 30 facets. Step two is to identify problems that may be associated with elevated scores on a specific domain spectrum. For example, the struggle an individual has with violence in social settings might be associated with his/her personality being high in antagonism. Step three is to determine if the impairment is clinically significant (i.e., does the problem require professional intervention). Step four, which is optional, is to match the given FFM profile, consisting of an individual's placement along each domain spectrum, quantitatively to profiles of specific PDs. This is done by correlating the FFM profile with a FFM description of a prototypical case of the specific PD (Samuel et al., 2011). It can also be accomplished by comparing the FFM profile to a prototypical profile which is a narrative description of each PD. These descriptions are 15-20 sentences long with no regulation or rule pertaining to which sentences are emphasized or used (Widiger \& Mullins-Sweatt, 2010). This last step is included as a secondary check of the clinician's diagnosis. Most clinicians skip this step because it is not necessary for diagnosing individuals and it can be time consuming (Widiger \& Mullins-Sweatt, 2010). 


\section{Benefits of Dimensional Models}

Like the previous categorical model, the FFM has both benefits and problems. Clinicians have reported high rates of clinical utility when diagnosing real clinical cases (Morey et al., 2014). It is operationally defined by ease of use, communication, and treatment planning (Widiger \& Mullins-Sweatt, 2010). The FFM is moderately easy to use. The structured interview for the FFM assesses individuals by domain first facilitating faster interviews. For example, if an individual rates high on agreeableness then there is no real need to assess for maladaptive variants of antagonism. It must be noted, however, that there are exceptions to this simplification (Widiger \& Mullins-Sweatt, 2010). Clinicians that trained with the categorical model of diagnosis, which include the majority of clinicians, are unfamiliar with the structure of the FFM and will have a more difficult time using it in a clinical setting (Skodol, 2012). The dimensional model provides a more precise and comprehensive quantitative description of PD diagnoses (Sprock, 2003), and a richness and subtlety in definition not previously seen (Clark, 2007) which leads to more meaningful communications between professionals and between clinicians and their patients. The improved PD description reduces the number of borderline cases seen by clinicians, allows for greater heterogeneity among patients (Widiger \& Mullins-Sweatt, 2010), and lowers comorbidity rates (Sprock, 2003). For treatment planning, clinicians have reported the domains and facets of the FFM to be highly useful because they facilitate the creation of more specific treatment plans. Additionally, the presence of normal, adaptive personality traits may assist clinicians with treatment planning (Widiger \& Mullins-Sweatt, 2010) by allowing them to assess their patients holistically and create a more specific treatment option. 


\section{Problems with Dimensional Models}

One of the main obstacles facing dimensional diagnostic models of PDs is determining where the cutoff lies between a clinically significant disorder and a non-clinically significant disorder. Disorders that are considered clinically significant receive an official diagnosis and are determined to be in need of treatment while a non-clinically significant disorder will not receive a diagnosis or treatment. The determination of this virtual line for pathology and what requires treatment and what does not, has minimal empirical evidence supporting its location along the dimensional spectrum (Berghuis et al., 2012). Another difficulty pertaining to dimensional models is the wide variety of the models themselves. The three models described earlier (the FFM, the SNAP, and the CTCI) vary significantly in diagnostic criteria from trait scales to temperament scales (Morey et al., 2007; Sprock, 2003; Widiger \& Mullins-Sweatt, 2010).

Dimensional measurements vary substantially and have been the focus of much recent research on PDs (Sprock, 2003). Most dimensional models employ a measurement tool, usually a self-report questionnaire, that is unique to their model. The FFM uses the NEO PI-R (Neuroticism, Extroversion, Openness Personality Inventory Revised) and the SIFFM (Structured Interview for the FFM) to assess individuals (Berghuis et al., 2012; Samuel et al., 2011). The Neo PI-R is a self-report measure consisting of 240 statements that are answered on a 5-point Likert scale (e.g., 1-not like me to 5-very like me) and the SIFFM is a guided interview used to assess the same basic domains and facets of the FFM. The SIFFM is the only interview measure, instead of a self-report measure, that has been created for the FFM (Samuel et al., 2011). Meanwhile, the Livesley's Adaptive Failure Model uses the General Assessment of Personality Disorder (GAPD) to define the core components of personality pathology. The GAPD is a self-report measurement that consists of 142 questions rated on a 5-point Likert scale 
(Berghuis et al., 2012). The measurements described above are just a few of the dimensional measurements that have been created for dimensional models of PDs. A unique measurement tool for each model shows an uncertainty among professionals of how to most effectively and accurately measure PDs dimensionally.

\section{Hybrid Model}

A hybrid model is made up of dimensions that are based on categories (Sprock, 2003) and functions as a compromise between a trait based system (i.e., dimensional model) and the polythetic categorical system (Yam \& Simms, 2014). An alternative hybrid model for PDs was added to section III of the DSM-5 although the official diagnostic model for PDs, located in section II of the DSM-5, is the traditional categorical model. The Axis organization of the DSMIV-TR was removed from the DSM-5 and mental disorders were organized into sections. Section II of the DSM-5 is for the official diagnostic criteria and codes for all mental disorders including PDs, while section III of the DSM-5 is for emerging measures and models (Krueger and Markon, 2014).

\section{DSM-5 Hybrid Model}

The alternative hybrid model of the DSM-5, hereafter referred to as DSM-5 hybrid model, includes six PD categories defined by a specific pattern of impairments and traits (APA, 2013), and a seventh PD classification for individuals who do not meet a specific category's criteria. The DSM-5 hybrid model includes eight changes to the original categorical model and its diagnostic constructs (Porter \& Risler, 2014). Some of the changes were broad and applied to all PDs while others were specific to a particular PD. 
First, four of the PD categories were removed. These categories included the Paranoid, Schizoid, Histrionic, and Dependent PD categories, leaving the Schizotypal, Antisocial, Borderline, Narcissistic, Avoidant, and Obsessive-Compulsive PD categories. This action was taken based on the rationale that there were minimal empirical studies focused specifically on those categories and the trait compositions were too simplistic to be included (Porter \& Risler, 2014; Skodol, 2012). Second, a functional impairment severity rating scale was added. The Levels of Personality Functioning Scale (LPFS) is a five-point rating scale that specifies the type and degree of functional impairment of an individual. The scale rates from 0 (no impairment) to 4 (major impairment) in all of the following areas: identity, self-direction, empathy, and intimacy (Krueger et al., 2014; Porter \& Risler, 2014). Third, twenty-five pathological personality trait descriptors were added and organized into five broad domains (later referred to as Criterion B). Forth, a new category, called "PD-trait specified," was add to the model for all the PDs that are not delineated as one of the other specific categories.

The DSM-5 hybrid model eliminated the strict temporal stability criterion which makes for a more accurate portrayal of PDs, and is supported by empirical evidence that shows a fluctuation in the expression of symptoms across time (Porter \& Risler, 2014). The Axis organization was also removed from the DSM so the Axis I exclusions were no longer necessary and therefore discarded. Also eliminated was the requirement that a patient have been previously diagnosed with a conduct disorder for them to qualify for an antisocial PD diagnosis. This makes the PD categories more consistent across the board (Porter \& Risler, 2014). Finally, it introduced a dimensional conceptualization of PDs which will be further discussed later. 


\section{PD Diagnostic Criteria for the DSM-5 Hybrid Model}

A PD diagnosis in the DSM-5 hybrid model is an amalgamation of two main assessments: impairments in personality functioning (self and interpersonal) (Criterion A) and descriptions of the associated pathological traits (Criterion B) (Skodol, 2012; Wright et al., 2012). Criterion A distinguishes diagnostic severity (i.e., the overall level of personality pathology) and is assessed with the Levels of Personality Functioning Scale (LPFS) previously described (Krueger et al., 2014). This scale rates an individual along two domains, self, described as identity and self-directed behavior, and interpersonal, described as capacity for intimacy and empathy. There are two basic functions of Criterion A (Krueger et al., 2014). The first is to describe general PD or what is universal amongst PDs. The second is to articulate symptomatic features of PDs in terms of a deficit in one of the LPFS domains. Criterion B captures the individual differences in the style of PD manifestation (Krueger et al., 2014) and is evaluated with a tool called the PID-5 that is further explained later. There are five broad domains that are based on 25 specific traits. These domains are: negative affectivity vs emotional stability, detachment vs extraversion, antagonism vs agreeableness, disinhibition vs conscientiousness, and psychoticism (i.e., aggressiveness and hostility) vs lucidity (i.e., clear thinking) (Krueger et al., 2014).

The Personality Inventory for the DSM-5 (PID-5) is a 220 item assessment that measures specific elements of maladaptive personality (Krueger \& Markon, 2014). It measures 25 specific elements itemized within five broad domains that resemble the Big 5 model of personality and the FFM (Wright et al., 2012). The five domains are: negative affect, detachment, antagonism, disinhibition, and psychoticism. The PID-5 domains closely match those of the DSM-5 hybrid 
model Criterion B even though this assessment was derived independently of the model (Kreuger \& Markon, 2014; Wright et al., 2012).

When the Criterion A and B assessments are both considered, the result is a diagnosis of one of the seven possible PD categories retained by the DSM-5 hybrid model (Anderson et al., 2014). Specifically, Criterion A reveals the presence of a disorder and evaluates the severity of that disorder, while Criterion B expounds on specific trait facets of the disorder and facilitates the assignment of a categorical PD diagnosis. All PDs display the same general criteria and only differ in the level and type of impairment expressed (Porter \& Risler, 2014).

\section{Benefits and Problems of the DSM-5 Hybrid Model}

Since the DSM-5 hybrid model is new to the field there has not been a significant amount of research conducted on it, and the research that has been done is contradictory. This causes the benefits and problems pertaining to the hybrid model of PDs to overlap substantially. Clinicians report that the DSM-5 hybrid model has high levels of clinical utility, is easily applied to patients, and makes communicating with patients less complicated (Krueger et al., 2014). The LPSF is rated as a valid predictive measure of personality pathology, and clinicians praise the inclusion of a severity rating (Krueger et al., 2014; Skodol, 2012). Within the DSM-5 hybrid model PDs are conceptualized dimensionally, which was listed in the "DSM-5 Hybrid Model" section as one the eight ways the hybrid model changed from the categorical model. PDs are conceptualized by the unique difficulties in personality functioning of an individual and the specific patterns of their pathological traits While these are important benefits, the DSM-5 hybrid model has also been the target of substantial criticism. Several members of the work group that designed the model were not satisfied with the final product because the general criteria lacked empirical 
support (Porter \& Risler, 2014). Also the constructs of the DSM-5 hybrid model are far too complex to be practically relevant to clinicians resulting in low clinical utility (Porter \& Risler, 2014), which is an example of the contradictory research pertaining to the DSM-5 hybrid model. The two models that are combined to create the hybrid model utilize opposite frameworks. The categorical expresses the constructs as being discontinuous with one another while the dimensional framework expresses the constructs as being continuous throughout (Porter \& Risler, 2014). These competing construct frameworks create complex constructs that are difficult to use in a clinical setting. It is acknowledged that many of the proposed changes have not been tested and have not proven to be an advantage over the current system (Gunderson, 2013).

\section{Other Significant Influences}

The diagnostic model included in the DSM is primarily used for diagnostic and treatment purposes, however, it is also used to communicate with insurance companies. Insurance companies use a code system to determine what treatments are covered within their policies. A patient is required to have a diagnostic code in order to receive insurance coverage for their treatment and the code must correspond to their diagnosed disorder. This system is set up to require a diagnostic code and therefore a DSM diagnosis before insurance companies will cover the cost of treatment; although some exceptions may apply. However, the Health Insurance Portability and Accessibility Act (HIPAA) requires insurance companies to use the U.S. Clinical Modifications of the World Health Organization's International Classification of Diseases ninth edition (ICD-9-CM) diagnostic codes not the DSM-IV-TR diagnostic codes for mental disorders. As a result most insurance companies are likely to accept only the ICD-9-CM diagnostic codes (Covered Diagnoses \& Crosswalk of DSM-IV Codes to ICD-9-CM Codes, 2002). The APA 
published a "crosswalk" to help clinicians match their DSM-IV-TR diagnoses and codes to those of the ICD-9-CM for insurance purposes. The DSM-5, however, does not use its own coding system, and is completely compatible with the HIPAA required ICD-9-CM now ICD-10-CM diagnostic codes (Insurance Implications of DSM-5, 2013). The code system used by insurance companies influences the type of diagnostic model used to diagnose PDs and other mental disorders. The categorical model allows for easy inclusion of the diagnostic codes required by the insurance companies. Each specific PD diagnosis can correspond to a different diagnostic code. It is currently unclear as to how the dimensional model can incorporate diagnostic codes required by insurance companies when their diagnoses are patient specific.

Insurance coverage possibilities is one of the political influences the APA must consider before they make the final decision as to which diagnostic model is published in the DSM. The paradigm shift from categorical to dimensional is dictated by the diagnostic model included in the DSM, therefore the shift is significantly influenced by the APA Board of Trustees. The DSM-5 has two parallel diagnostic models for PDs, the categorical model in section II and the hybrid model in section III. This is a result of the power structure and process used by the APA when creating a new edition to the DSM (Krueger \& Markon, 2010). The group of experts assigned to revise the PD section of the DSM-5 approved the inclusion of the hybrid model in Section II of the DSM, however, it was not included in Section II (Krueger et al., 2014), but in Section III as an emerging measure and model. The APA Board of Trustees had the final authority to decide the content of the DSM-5. They chose to retain the categorical model of the DSM-IV-TR for the sake of continuity with current clinical practice (Krueger \& Markon, 2014) and because of the large amounts of criticism the hybrid model received (Section III, 2013; Personality Disorders, 2013). The APA included the new model in Section III in hopes that it 
would encourage research to be conducted regarding this model given that it addresses the existing issues of the categorical model (Krueger \& Markon, 2014).

\section{DISCUSSION}

\section{Comparing the Models}

All three models have their strengths and weaknesses. The clinical utility rating of each model is dependent on what type of clinician is rating the model (e.g., psychiatrist, clinical psychologist, and counselor) and the type of cases they are using the model to diagnose. The DSM-IV-TR is rated higher in clinical utility by all levels of clinicians when diagnosing prototypical cases (Morey et al., 2014). This is to be expected seeing as prototypical cases are created based on DSM-IV-TR diagnostic criteria. Clinicians have been utilizing the categorical model in the DSM-IV-TR from the time it was originally published in the DSM-III in 1980 and thus have had about 35 years to practice and become comfortable with this model and its diagnostic criteria. Both the dimensional model and the hybrid model are relatively new to the field; therefore, clinicians are unfamiliar with these models and have had a limited amount of time to learn how to use them (Skodol, 2012). Lower clinical utility ratings can be expected especially for prototypical cases. When diagnosing actual cases seen in a clinical setting, the dimensional model was rated the most clinically useful by non-psychiatrists, while psychiatrists rated the DSM-IV-TR model as being more clinically useful (Morey et al., 2014). Psychiatrists generally emphasize diagnostics and they typically rate the simplest diagnostic model as the better model. This could account for the difference in clinical utility ratings between psychiatrists and non-psychiatrists. The difference could also be due to the familiarity and novelty issues mentioned above. 
Dimensional models, specifically the FFM, have proven to reliably describe all of the DSM-IV-TR PDs (Samuel et al., 2011; Widiger \& Mullins-Sweatt, 2010) in terms of behavioral traits measured within the model. The PD traits of the DSM-5 hybrid model have been shown to generally predict the PD criterion for the DSM-IV-TR PDs (Yam \& Simms, 2014). Both the FFM and the DSM-5 hybrid model have shown that they are capable of reliably, but not perfectly, reproducing the DSM-IV-TR categorical model. These models have typically been rated based on this ability to recreate the DSM-IV-TR categorical diagnoses. This replication of the DSM-IV-TR PD diagnostic constructs is not a decisive measure of the quality of the alternative models because the categorical system is full of problems (Krueger et al., 2014). The categorical model has not had any significant revisions made in the 35 years since its original publication in the DSM-III, despite the fact that there are numerous issues associated with it and empirical evidence supports a dimensional model. It would be exceedingly beneficial to focus on moving toward a valid, empirically based system rather than allowing the traditional method to prevent progress. Many professionals criticize the continued use of categories to describe PDs when there is sufficient evidence showing the framework to be inaccurate (Porter \& Risler, 2014).

\section{Considering the Paradigm Shift}

An ideal diagnostic model of PDs would be reliable and useful to clinicians. It would allow for effective communication, provide a useful structure for case conceptualization, and facilitate treatment planning (Sprock, 2003). Essentially, it must provide a framework for assessing, diagnosing, summarizing, and communicating (e.g., noting in a chart) a patient's personality traits, as well as the gradation of severity, that are significantly important for 
prognosis and treatment planning in everyday psychiatric practice (Shedler et al., 2010). A model like this would convey a holistic, person-centered understanding of the patient to all relevant parties (Krueger et al., 2014). The coverage of the model, meaning the extent to which the model adequately represents and describes the conditions and symptoms that are frequently encountered by clinicians and studied by researchers, is important to consider when measuring the quality of the model (Clark, 2007).

The ideal model can be achieved by applying a dimensional diagnostic model for PDs. The dimensional model lessens the comorbidity and co-occurrence problems that were related to the DSM-IV-TR model significantly. This is accomplished by incorporating multiple dimensions on which individuals can vary (Krueger \& Markon, 2014; Skodol, 2003; Widiger \& MullinsSweatt, 2010) and providing a more precise comprehensive quantitative description of personality pathology (Sprock, 2003) which proves this model to be more useful for treatment planning (Widiger \& Mullins-Sweatt, 2010). Considering all the evidence, it can be concluded that a dimensional model does represent the theoretical framework of PDs more holistically than the other two main types of models.

Theoretically, a dimensional model should be utilized by the DSM. Changing from a categorical to dimensional diagnostic model of PDs in the DSM will begin a paradigm shift from a categorical to a dimensional conceptualization of all mental disorders. However, there is not enough empirical research on the practical uses of both the dimensional model and the hybrid model to claim one model is more advantageous than the others. There is also a resistance among some clinicians to any diagnostic changes because they are comfortable with the categorical model that they have been using for the last 35 years and they know how to get insurance companies to pay for treatments. Due to these barriers the APA was justified in their reluctance 
and decision not to change the official PD diagnostic criteria in the DSM-5. Such a change would have a domino effect and start a chain reaction of diagnostic transitions throughout the DSM. The implications of this change in diagnostic models of PDs are substantial. A decision to implement change based on the current amount of empirical research and with the resistance among clinicians is ill advised. It must be determined whether the dimensional model suits the diagnosis of PDs as well in a practical setting as it does a theoretical one. Training programs should also be implemented to assist clinicians with the vast differences in diagnostic styles of the dimensional model so they can become as comfortable with it as they are with the categorical model. Although the change from a categorical to a dimensional diagnostic model for PD is necessary the psychological community is not yet ready to embrace it.

\section{Limitations}

Although I have not used any of these models (i.e., categorical, dimensional, or hybrid) in a clinical setting and therefore have no practical experience using the models I do have a theoretical understanding of them. This limits my ability to refer any important practical nuances each model may contain. Another limitation of this thesis is the lack of empirical research that has been conducted on the theoretical and practical applications of the DSM-5 Section III hybrid model. The DSM-5 has only been available for two years and although a significant amount of research has been completed there is a need for a great deal more. Significant gaps exists in the empirical research pertaining to the practical application, in areas such as stigmatizing effects on patients, insurance coverage, and the long term effects of dimensional models on patient quality of life. Any claim of superiority among the diagnostic model types cannot be made. 


\section{Future Research}

To address some of the limitations of this thesis (i.e., the gaps in research on practical applications of dimensional models), future research efforts should include focusing on alternative diagnostic models (e.g., FFM or DSM-5 Hybrid Model) and their effects on stigmatization. Research should also focus on the effects a dimensional PD model will have on the relationship between clinicians and insurance companies. Given that most insurance plans require the DSM or ICD statistical code of the diagnosis before they will cover treatment costs, it is important that research be done on possible financial and coverage repercussions of the dimensional model. Future research on the long term effects of the alternative diagnostic models is also needed. The question of whether these models actually work in practice to improve the patient's quality of life must be answered. This can only be done by using the alternative models and measuring their treatment outcomes. Only when these gaps have been filled can a paradigmatic shift from a categorical to a dimensional conceptualization of PDs, and eventually all psychological disorders, occur. 


\section{References}

Anderson, Jaime, Stephen Snider, Martin Sellbom, Robert Krueger, and Christopher Hopwood. "A Comparison of the DSM-5 Section II and Section III Personality Disorder Structures."

Psychiatry Research 216, no. 3 (May 30, 2014): 363-72. doi:10.1016/j.psychres.2014.01.007.

American Psychiatric Association. (2000). Diagnostic and statistical manual of mental disorders (4th ed., text rev.). Washington, DC: Author.

American Psychiatric Association. (2013). Preface. In Diagnostic and statistical manual of mental disorders (5th ed.). Washington, DC: Author.

American Psychiatric Association. "Insurance Implications of DSM-5." Psychiatric News 48, no. 9 (May 3, 2013): 1-1. doi:10.1176/appi.pn.2013.5a27.

American Psychiatric Association. "Personality Disorders" Psychiatric News 48, no. 9 (May 3, 2013): $1-2$.

American Psychiatric Association. "Section III" Psychiatric News 48, no. 9 (May 3, 2013): 1-3.

Arbisi, Paul A. "Introduction to the Special Section on the Personality Psychopathology Five (PSY-5) and DSM-5 Trait Dimensional Diagnostic Systems for Personality Disorders: Emerging Convergence." Journal of Personality Assessment 96, no. 2 (March 4, 2014): 117-20. doi:10.1080/00223891.2013.866571.

Berghuis, Han, Jan H. Kamphuis, and Roel Verheul. "Core Features of Personality Disorder: Differentiating General Personality Dysfunctioning from Personality Traits." Journal of Personality Disorders 26, no. 5 (2012): 704-16.

Blashfield, Roger K., Jared W. Keeley, Elizabeth H. Flanagan, and Shannon R. Miles. "The Cycle of Classification: DSM-I Through DSM-5.” Annual Review of Clinical Psychology 10, no. 1 (2014): 25-51. doi:10.1146/annurev-clinpsy-032813-153639. 
Clark, Lee Anna. "Assessment and Diagnosis of Personality Disorder: Perennial Issues and an Emerging Reconceptualization.” Annual Review of Psychology 58, no. 1 (2007): 227-57. doi:10.1146/annurev.psych.57.102904.190200.

"Covered Diagnoses \& Crosswalk of DSM-IV Codes to ICD-9-CM Codes." Http://www.apapracticecentral.org. Accessed May 1, 2015. http://www.apapracticecentral.org/reimbursement/billing/icd-9-cm.aspx.

Grant, Bridget F., Frederick S. Stinson, Deborah A. Dawson, S. Patricia Chou, and W. June Ruan. "Co-Occurrence of DSM-IV Personality Disorders in the United States: Results from the National Epidemiologic Survey on Alcohol and Related Conditions." Comprehensive Psychiatry 46, no. 1 (2005): $1-5$.

Gunderson, John. "Understanding the Speed of Change: Rejoinder for 'Seeking Clarity for Future Revisions of the Personality Disorders in DSM-5'." Personality Disorders: Theory, Research, and Treatment 4, no. 4 (October 2013): 381. doi:http://dx.doi.org/10.1037/per0000052.

Hinshaw, S., Cicchetti, D., \& Toth, S. (2006). What Is Mental Disorder and What Is Stigma? In Mark of Shame : Stigma of Mental Illness and an Agenda for Change (pp. 3-27). Oxford University Press.

Krueger, Robert F., Christopher J. Hopwood, Aidan G. C. Wright, and Kristian E. Markon. "DSM-5 and the Path Toward Empirically Based and Clinically Useful Conceptualization of Personality and Psychopathology." Clinical Psychology: Science and Practice 21, no. 3 (2014): 245-61. doi:10.1111/cpsp.12073.

Krueger, Robert F., and Kristian E. Markon. "The Role of the DSM-5 Personality Trait Model in Moving Toward a Quantitative and Empirically Based Approach to Classifying Personality and 
Psychopathology." Annual Review of Clinical Psychology 10, no. 1 (2014): 477-501. doi:10.1146/annurev-clinpsy-032813-153732.

Morey, Leslie C., Christopher J. Hopwood, John G. Gunderson, Andrew E. Skodol, M. Tracie Shea, Shirley Yen, Robert L. Stout, et al. "Comparison of Alternative Models for Personality Disorders." Psychological Medicine 37, no. 07 (July 2007): 983-94. doi:10.1017/S0033291706009482.

Morey, Leslie C., Andrew E. Skodol, and John M. Oldham. "Clinician Judgments of Clinical Utility: A Comparison of DSM-IV-TR Personality Disorders and the Alternative Model for DSM-5 Personality Disorders.” Journal of Abnormal Psychology 123, no. 2 (May 2014): 398-405. doi:http://dx.doi.org/10.1037/a0036481.

Porter, Jeffrey S., and Edwin Risler. "The New Alternative DSM-5 Model for Personality Disorders Issues and Controversies." Research on Social Work Practice 24, no. 1 (January 1, 2014): 50-56. doi:10.1177/1049731513500348.

Samuel, Douglas B., Maryanne Edmundson, and Thomas A. Widiger. "Five Factor Model Prototype Matching Scores: Convergence Within Alternative Methods.” Journal of Personality Disorders 25, no. 5 (October 2011): 571-85. doi:10.1521/pedi.2011.25.5.571.

Shedler, Jonathan, Aaron Beck, Peter Fonagy, Glen O. Gabbard, John Gunderson, Otto Kernberg, Robert Michels, and Drew Westen. "Personality Disorders in DSM-5." American Journal of Psychiatry 167, no. 9 (September 2010): 1026-28. doi:10.1176/appi.ajp.2010.10050746.

Skodol, Andrew E. "Personality Disorders in DSM-5.” Annual Review of Clinical Psychology 8, no. 1 (2012): 317-44. doi:10.1146/annurev-clinpsy-032511-143131.

Sprock, June. "Dimensional Versus Categorical Classification of Prototypic and Nonprototypic Cases of Personality Disorder." Journal of Clinical Psychology 59, no. 9 (September 2003): 991-1014. 
Tackett, Jennifer L., Amy L. Silberschmidt, Robert F. Krueger, and Scott R. Sponheim. “A Dimensional Model of Personality Disorder: Incorporating DSM Cluster A Characteristics.," 2009. http://psycnet.apa.org/journals/per/S/1/27/.

Widiger, Thomas A., and Stephanie N. Mullins-Sweatt. "Clinical Utility of a Dimensional Model of Personality Disorder." Professional Psychology: Research and Practice 41, no. 6 (December 2010): 488-94. doi:http://dx.doi.org/10.1037/a0021694.

Wright, Aidan G. C., Katherine M. Thomas, Christopher J. Hopwood, Kristian E. Markon, Aaron L. Pincus, and Robert F. Krueger. "The Hierarchical Structure of DSM-5 Pathological Personality Traits." Journal of Abnormal Psychology 121, no. 4 (November 2012): 951-57. doi:http://dx.doi.org/10.1037/a0027669.

Yam, Wern How, and Leonard J. Simms. "Comparing Criterion- and Trait-Based Personality Disorder Diagnoses in DSM-5." Journal of Abnormal Psychology 123, no. 4 (November 2014): 802-8. doi:http://dx.doi.org/10.1037/a0037633. 